\title{
Amiga 1000 hardware timing and reaction-time key interfacing
}

\author{
RICHARD D. WRIGHT \\ The University of Western Ontario, London, Ontario, Canada
}

\begin{abstract}
A method of hardware reaction timing with millisecond accuracy, using one of the Amiga's CIA 8520 chips, is described. The registers of this chip can be set to enable cascaded timing that functions independently of the CPU and, thereby, avoids the problems of software timing in a multitasking environment. In addition, the interfacing of a pair of reaction-time keys to one of the Amiga's game controller connectors and a program for polling this port for keypresses are described.
\end{abstract}

The Amiga is an impressive low-price graphics machine. Some of the features that make it an attractive research tool for investigators in such fields as visual psychophysics are its processing speed, the large number of colors it can display $(4,096)$, and its capacity to produce displays with four different screen resolutions. It can also carry out many more simultaneous, dynamic changes within a displayed image than has been possible with the previous generation of microcomputers (see Williams, Edwards, \& Robinson, 1985, for a review of the Amiga). The speed at which graphics operations take place is made possible by the Motorola 68000 chip, which allocates different tasks to three other custom chips. In this multitasking environment, the CPU is usually free to do various system maintenance tasks while the graphics coprocessors carry out tasks for which they were specifically designed.

The obvious advantage of multitasking is an increase in overall processing speed. However, with a multitasking environment, the measurement of temporal intervals is more complex than is typically the case with microcomputers, particularly for software timing methods. This complexity is a consequence of cycle-stealing by the graphics chips. In particular, the clock speed of the 68000 is $7.159 \mathrm{MHz}$ but the absolute time required for operations to be carried out by this chip depends on the number of machine cycles "stolen" by its companion chips performing other concurrent tasks. This could disrupt the accuracy of software timers (see, e.g., Price, 1979), which work by decrementing a counter value a particular number of times.

\section{TIMING WITH THE 8520 CHIPS}

A reliable alternative to software timing is the use of on-board hardware clocks. These are available in the Am-

The writing of this report was supported by an NSERC postgraduate scholarship awarded to the author and by NSERC Grant No. A2600 awarded to Zenon Pylyshyn. I am grateful to Jim Clark, Mike Dawson, and Zenon Pylyshyn for their help. Requests for reprints should be sent to Richard D. Wright, Centre for Cognitive Science, Department of Psychology, The University of Western Ontario, London, Ontario, Canada N6A 5C2. iga's two CIA (Complex Interface Adapter) 8520 chips. The 8520 s are similar to the CIA 6526 s of the Commodore 64, described by Kallman (1986). Each 8520 chip has two timers that have clock speeds of $716 \mathrm{KHz}$ and can be programmed as one-shot, continuous, or cascaded timers. The $8520 \mathrm{~B}$ timers are used continuously by the system, but the $8520 \mathrm{~A}$ timers are available if complex graphics operations or serial communications are not in process during the timed interval. In this article, I describe the steps required to program the $8520 \mathrm{~A}$ chip as a cascaded reaction timer, and to interface reaction-time keys with this timer through game controller connector \#2 (on the right side of the computer).

\begin{tabular}{|c|c|c|c|c|}
\hline 1 & & & & *Reaction T1me Program \\
\hline 2 & $13 F C 0000$ & $\mathrm{OOBF}$ & EE01 & $\begin{array}{l}\text { MOVE. B } \# \$ 00, \$ B F E E 01 \\
\text { (Set CTLA to O) }\end{array}$ \\
\hline 3 & $13 F C 0000$ & OOBF & EF01 & $\begin{array}{l}\text { MOVE. B } \# \$ 00, \$ B F E F 01 \\
\text { (Set CTLB to O) }\end{array}$ \\
\hline 4 & $13 F C$ OOCC & $\mathrm{OOBF}$ & E401 & $\begin{array}{l}\text { MOVE.B \#\$CC, } \$ B F E 401 \\
(\text { Set TALO to CC) }\end{array}$ \\
\hline 5 & $13 F C 0002$ & OOBF & E501 & $\begin{array}{l}\text { MOVE.B \#\$02, \$BFE501 } \\
\text { (SOt TAHI to } 2 \text { ) }\end{array}$ \\
\hline 6 & 13FC OOFF & OOBF & E601 & $\begin{array}{l}\text { MOVE. B } \# \$ F F, \$ B F E 601 \\
\text { (Set TBLO to FF) }\end{array}$ \\
\hline 7 & $13 \mathrm{FC} \mathrm{OOFF}$ & OOBF & E701 & $\begin{array}{l}\text { MOVE. B \#\$FF, \$BFE701 } \\
\text { (Set TBHI to FF) }\end{array}$ \\
\hline 8 & 13FC 0041 & OOBF & EFO1 & $\begin{array}{l}\text { MOVE.B \#\$41, \$BFEFO1 } \\
\text { (Start T1mer B) }\end{array}$ \\
\hline $\boldsymbol{9}$ & $13 \mathrm{FC} 0001$ & OOBF & EE01 & $\begin{array}{l}\text { MOVE. B } \# \$ 01, \$ B F E E 01 \\
\text { (Start T1mer A) }\end{array}$ \\
\hline 10 & $3039000 \mathrm{~F}$ & F016 & & $\begin{array}{l}\text { MOVE.W \$DFFO16, DO } \\
\text { (LOad POTINP 1nto DO) }\end{array}$ \\
\hline 11 & $323 \mathrm{C} 5500$ & & & $\begin{array}{l}\text { MOVE.W \#\$5500,D1 } \\
\text { (Load } 5500 \text { 1nto D1) }\end{array}$ \\
\hline 12 & $\mathrm{~B} 240$ & & & $\begin{array}{l}\text { CMP DO.D1 } \\
\text { (Compare DO \& D1) }\end{array}$ \\
\hline 13 & $67 F_{2}$ & & & $\begin{array}{l}\text { BEQ loop } \\
\text { (Branch 1f equal) }\end{array}$ \\
\hline 14 & $13 \mathrm{FC} 0000$ & OOBF & EEO1 & $\begin{array}{l}\text { MDVE. B \#\$00, \$BFEE01 } \\
\text { (Stop T1mer A) }\end{array}$ \\
\hline 15 & $13 \mathrm{FC} 0008$ & OOBF & EFO1 & $\begin{array}{l}\text { MOVE.B } \# \$ 08, \$ B F E F 01 \\
(\text { Re-enable } I / 0)\end{array}$ \\
\hline 16 & $4 E 74$ & & & RTS \\
\hline 17 & & & & END \\
\hline
\end{tabular}

Figure 1. An assembly listing of a 68000 machine language program for setting the $8520 \mathrm{~A}$ registers to enable cascaded timing and for detecting reaction-time keypresses. 
The $8520 \mathrm{~A}$ chip contains 168 -bit registers, 6 of which must be set to enable cascaded timing. Figure 1 is an assembly listing of a program for setting these registers. In order to cascade Timer A and Timer B, Control Register B (\$BFEF01) must have bits 0 and 6 set high (i.e., set to $\$ 41$; see line 8 of Figure 1). Note that the $\$$ prefix denotes hexadecimal numbers. To start and stop Timer A underflow pulses, the zero bit of Control Register A (\$BFEE01) must be set high (\$01) and low (\$00), respectively. In addition, the Timer A Low (\$BFE401) and Timer A High (\$BFE501) registers must be set to $\$ C C$ and $\$ 02$, respectively. With these latch values, an underflow pulse is created by Timer A each millisecond. When the 8520A is set for cascaded timing, each Timer A underflow pulse increments the values of the Timer B Low (\$BFE601) and Timer B High (\$BFE701) registers, both of which are initially set to \$FF. For the benefit of those who do not have access to an assembler, Figure 2 is a listing of an AmigaBASIC program with a machine language subroutine to set the $8520 \mathrm{~A}$ registers and to poll game controller connector \#2 for a reaction-time keypress.

Some caveats are in order for those using the $8520 \mathrm{~A}$ for timing. Timer $A$ is available unless serial communications are being carried out. Timer $\mathbf{B}$ is available unless the blitter device is being synchronized to the video beam but is otherwise available when this graphics operation is not in progress. In addition, disk $\mathrm{I} / \mathrm{O}$ is disabled when using the $8520 \mathrm{~A}$ as a cascaded timer, because the fourth bit of Control Register B (\$BFEF01) is not set high. One solution to this problem is to reset this register to $\$ 8 \mathrm{im}$ mediately after the timed interval has elapsed (see line 15 in Figure 1). This re-enables disk I/O but does not solve the problem for those who must invoke I/O opera-

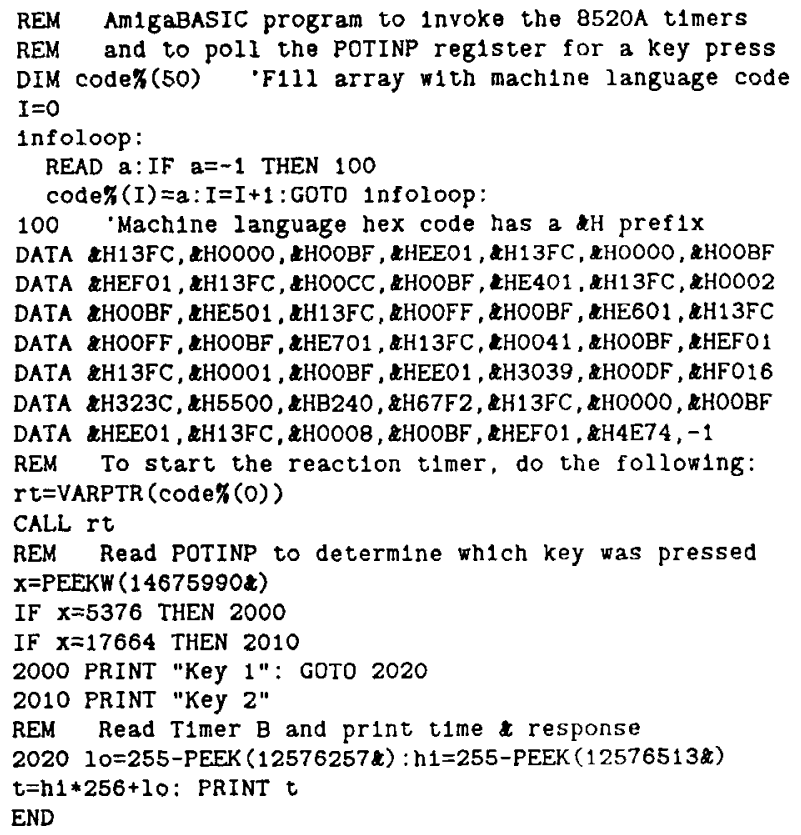

Figure 2. An AmigaBASIC program for loading and calling the machine language reaction-time subroutine in Figure 1.

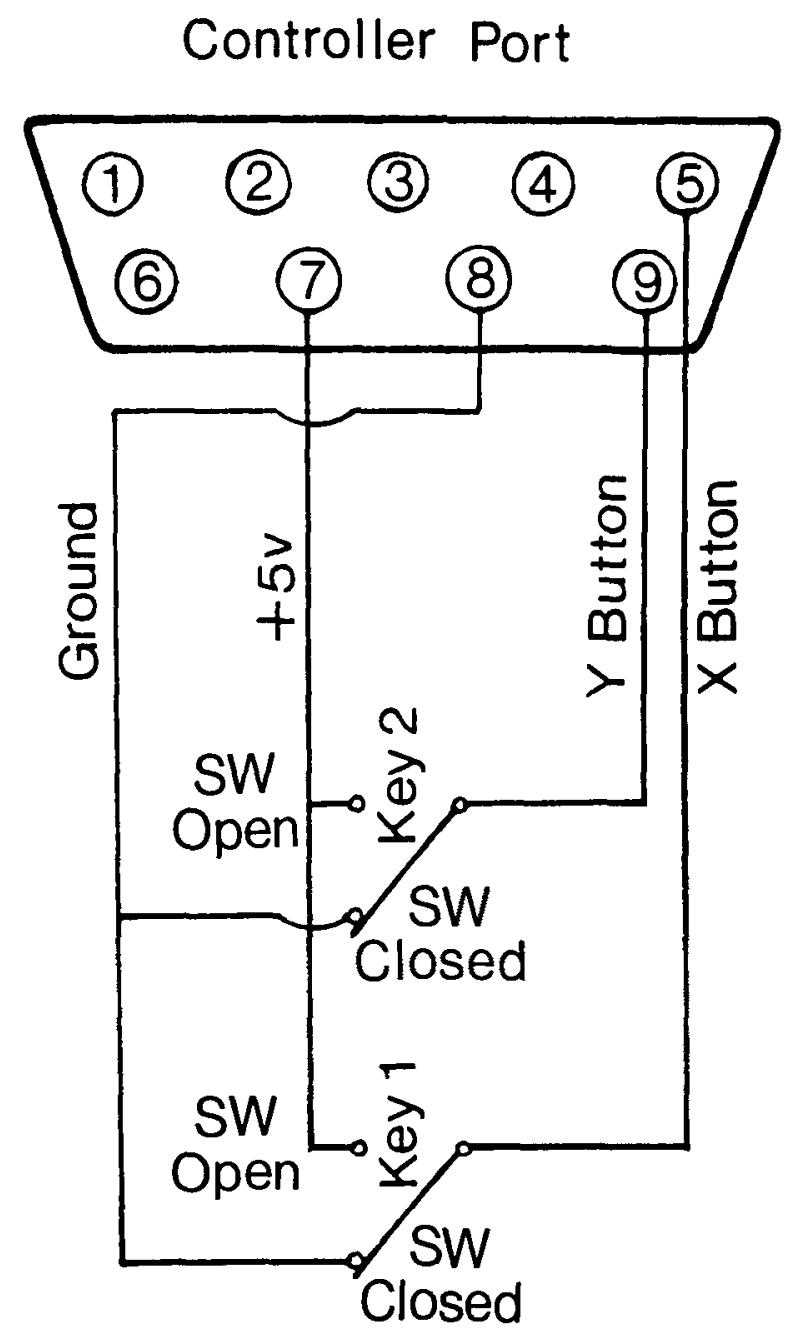

Figure 3. Schematic diagram of the reaction-time keys interfaced to game controller connector \#2. The $X$ and $Y$ buttons are normally set high $(+5 \mathrm{~V})$ but go low $(0 \mathrm{~V})$ when keypresses short pins 5 and 9 to ground (pin 8).

tions during the timed interval. Finally, care should be taken not to write to disk immediately before calling the timer. Precise control of the time that disk-writes occur is not possible because this is a low-priority operation in the Amiga multitasking environment. Therefore, a diskwrite invoked immediately before the timer is called may be delayed by the system until after timing operations have begun and disk $\mathrm{I} / \mathrm{O}$ is disabled.

\section{INTERFACING REACTION TIME KEYS}

The Amiga controller connectors can be programmed to interface with custom-built controllers, such as reactiontime keys. Figure 3 is a schematic wiring diagram for interfacing two keys with controller connector \#2 (a DB9 male). Pins 5 and 9 of this port normally receive $+5 \mathrm{~V}$ (switch open) but are set to $0 \mathrm{~V}$ when keypresses occur (switch closed).

By setting the POTGO Register (\$DFF034) to $\$ 0$, game controller connector \#2 is programmed to operate like a 
parallel port with two data lines (pins 5 and 9). The occurrence of keypresses can be read by polling the POTINP Register (\$DFF016). In particular, the default value (when a keypress has not yet occurred since setting POTGO) of this register is $\$ 5500$ (21760 in decimal). When key 1 is pressed, the POTINP register is set to \$44F4 (17664 in decimal), and when key 2 is pressed, this register is set to $\$ 14 \mathrm{~F} 4$ (5376 in decimal). The program in Figure 1 polls POTINP for a keypress and stops the cascaded timer when one occurs. If more than two keys are required, it is possible to interface the reactiontime keys to the Amiga's parallel port and to poll the data register of this port for keypresses (see p. 8-25 of the Amiga Hardware Manual, Commodore-Amiga, 1985) for information on programming this port). Polling either the game connector or the parallel port for a response is preferable to polling the keyboard because the keyboard buffer is scanned only at 16.67 -msec intervals. Hence, using the keyboard to stop the clock introduces an additional $16.67 \mathrm{msec}$ of variability into the reaction-time measure.
Note that when using this method of reaction timing, keypresses are not detected independently in the background. The POTINP register must be polled during the timed interval. However, if one does not require a timer that operates while complex graphics operations, disk $\mathrm{I} / \mathrm{O}$, or serial communications are being carried out, the use of the $8520 \mathrm{~A}$ timers is reliable and avoids the complexities inherent in Amiga software timing.

\section{REFERENCES}

Commodore-Amiga, Inc. (1985). Amiga hardware manual. West Chester, PA: Author.

KALLMAN, K. J. (1986). A Commodore 64-based experimental psychology laboratory. Behavior Research Methods, Instruments, \& Computers, 18, 222-227.

Pruce, J. M. (1979). Software timing for 6500 series microcomputers. Behavior Research Methods \& Instrumentation, 11, 568-571.

Williams, G., Edwards, J., \& Robinson, P. (1985). The Amiga personal computer. Byte, 10(8), 83-100.

(Manuscript received August 1, 1986; accepted for publication August 13, 1986.) 